\title{
No Significant Displacement of Basal Brain Structures upon Head Movement: Kinematic MRI Morphometry Relevant to Neuroendoscopy
}

\author{
Avril Horsburgh $^{1}$ Ramez W. Kirollos ${ }^{2}$ Tarik F. Massoud ${ }^{1,3}$ \\ ${ }^{1}$ Section of Neuroradiology, Addenbrooke's Hospital, Cambridge, \\ United Kingdom \\ 2 Department of Neurosurgery, Addenbrooke's Hospital, Cambridge, \\ United Kingdom \\ ${ }^{3}$ Department of Radiology, University of Cambridge, Cambridge, \\ United Kingdom \\ Address for correspondence Tarik F. Massoud, MD, PhD, Department \\ of Radiology, Section of Neuroradiology, Laboratory of Experimental \\ and Molecular Neuroimaging (LEMNI), Molecular Imaging Program at \\ Stanford (MIPS), Stanford University School of Medicine and Medical \\ Center, The James H. Clark Center, E153, 318 Campus Drive, East Wing, \\ First Floor Stanford, CA 94305-5427, United States \\ (e-mail: tmassoud@stanford.edu).
}

J Neurol Surg A 2014;75:98-103.

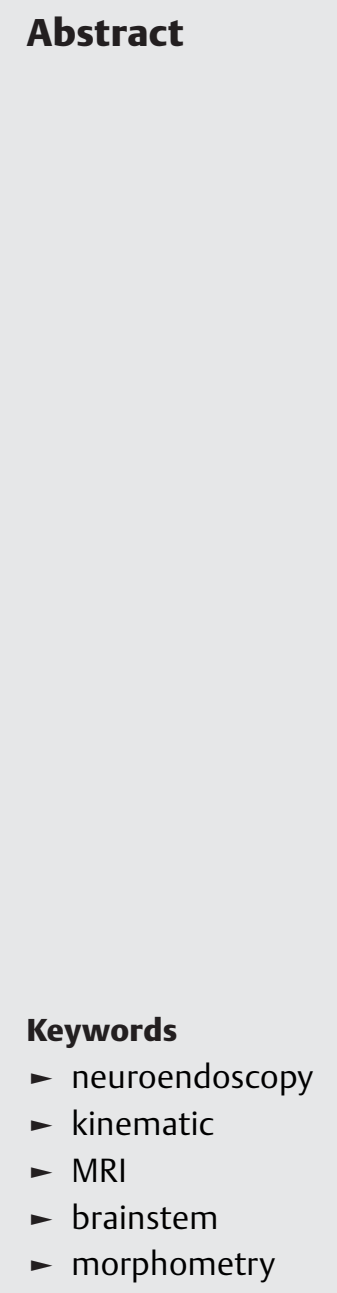

Background An appreciation of normal intracranial anatomy allows optimal planning of trajectories necessary for safe and effective neuroendoscopy. Little information exists on displacement of the caudal brain relative to the skull upon head movement; this could have important implications for planning and performance of neuroendoscopic procedures. We used kinematic magnetic resonance imaging (MRI) studies to examine the morphometric displacement and changing anatomical relationships between the clivus and basal brain structures, intracranial vessels, and subarachnoid spaces.

Patients We retrospectively analyzed 15 patients undergoing sagittal T2 kinematic MRI of the head and neck in modest flexion and extension. The angle between a horizontal axial reference plane and a line between the opisthion and the hard palate defined the degree of flexion and extension. We then measured in flexion and extension (1) the cervicomedullary angle (CMA), (2) displacement of the ventral surface of the brainstem (i.e., depth of the prepontine and premedullary cisterns), (3) total sagittal area of the combined suprasellar and ventral brainstem cisterns, and (4) the basilar tip to tuber cinereum distance.

Results Relative to neutral head position, a mean extension angle of -15.8 degrees was achieved in all 15 patients, and a mean flexion angle of +9.9 degrees was achieved in 6 patients. The mean CMA was 146 degrees in flexion and 158 degrees in extension. The mean reduction in prepontine and premedullary cistern depth was $0.7 \mathrm{~mm}$ and $0.5 \mathrm{~mm}$, respectively, upon flexion from extension. The combined area of suprasellar and ventral brainstem cisterns was minimally reduced from $402 \mathrm{~mm}^{2}$ in flexion to $399 \mathrm{~mm}^{2}$ in extension. The basilar tip did not move significantly from its position in flexion to extension, $5.3 \mathrm{~mm}$ to $5.2 \mathrm{~mm}$ respectively from the tuber cinereum.

Conclusion Kinematic MRI shows minimal brainstem-to-clivus displacement even within minor physiological changes in head flexion. Importantly, these movements are small and there is no significant shift in the position of the basilar tip in modest flexion or extension. These results should be useful for presurgical planning of optimal received

July 23, 2012

accepted after revision

December 20, 2012

published online

June 24, 2013 (c) 2014 Georg Thieme Verlag KG

Stuttgart · New York
DOI http://dx.doi.org/ 10.1055/s-0033-1342934. ISSN 2193-6315. 
patient positioning during neuroendoscopic procedures such as third ventriculostomy and the expanded endonasal transsphenoidal approach to the retroclival space.

\section{Introduction}

Therapeutic neuroendoscopy is an increasingly common option in minimally invasive neurosurgical practice. An appreciation of normal intracranial anatomy allows optimal planning of endoscope trajectories, which is necessary for safe and effective neuroendoscopic procedures.

To date, little information exists on the displacement of the caudal brain relative to the skull upon normal head movement. The relative displacement at the brain/skull boundary has often been considered in the context of studying traumatic brain injury, which entails relatively high differential velocities and impact between the two interfaces. To our knowledge, only one previous preliminary study has reported that noninvasive kinematic imaging analysis on five normal subjects in normal physiological conditions shows relative displacements of the brain with respect to skull as the head position changes. ${ }^{1}$

Potential displacement of basal brain structures upon head placement in different neurosurgical operative positions could have profound implications for the planning and performance of neuroendoscopic procedures. Thus, in this study, we hypothesized that upon placement of a patient's head in different positions in readiness for neuroendoscopic procedures, possible displacement of the brain relative to the skull might shift specific neuroendoscopic anatomical targets within the brain, rendering their location less predictable than anticipated. We therefore used kinematic magnetic resonance imaging (MRI) studies in subjects with no intracranial pathology to examine in detail the morphometric displacement and changing anatomical relationships upon head flexion and extension between the clivus and basal brain structures, intracranial vessels, and subarachnoid spaces-all anatomical structures relevant to a wide variety of neuroendoscopic procedures. Knowledge of predictable displacement of specific basal brain structures might also be of value in planning a priori the optimal endoscopic trajectories to reach particular intracranial locations.

\section{Patients}

All imaging was performed during routine neuroradiological work-up of patients based on clinical indications; no prospective imaging of normal volunteers was performed in this study. As such, and according to guidelines of the revised Declaration of Helsinki, this study reported the retrospective analysis of anonymized data (images) obtained from "routine sources" where consent of individual patients and ethical approval for research analysis was therefore not considered necessary. ${ }^{2,3}$

We retrospectively evaluated the studies of 15 patients undergoing sagittal kinematic MRI of the head and neck in modest flexion and extension, primarily for evaluation of degenerative changes in the mid and lower cervical spine. We used a 1.5T magnet (Signa HDx, GE Healthcare, Waukesha, Wisconsin, United States) and a standard posterior surface neck coil to obtain the images of the lower part of brain and cervical spine (cervical spine coil; GE Healthcare). Patients were scanned initially for imaging their cervical spine using standard T1- and T2-weighted static sagittal imaging and axial gradient echo imaging protocols. They were then scanned for kinematic imaging using a fast gradient echo multislice (GEMS) protocol: TR 3473, TE 1056, FOV $24 \mathrm{~cm}$, matrix $256 \times 128$, slice thickness $10 \mathrm{~mm}$, and slice gap $10 \mathrm{~mm}$. Two experienced neuroradiologists agreed by consensus and jointly analyzed the images on a Centricity ${ }^{\mathrm{TM}}$ PACS-IW review workstation (GE Healthcare). We first established the horizontal axial reference plane, as defined by Karhu et al, ${ }^{4}$ using electronic calipers on the midsagittal neutral head position images obtained. We found on all patients that this corresponded to a line parallel to the $\mathrm{C} 1 /$ C2 disc space as previously cross-referenced from axial images of the upper cervical spine. The angle between this line (line A in - Fig. 1) and another line between the opisthion and the hard palate (line B in - Fig. 1) defined the degree of flexion and extension: line $B$ was above line $A$ in extension, they overlapped in a neutral position, and B was below $A$ in flexion. ${ }^{4}$ In presentation of the data, the extension angle was prefixed by a minus sign and flexion angle by a plus sign relative to zero angle in the neutral position. Using the appropriate cursors on the review workstation for length, angle, or area enclosed within a tracing, we then measured

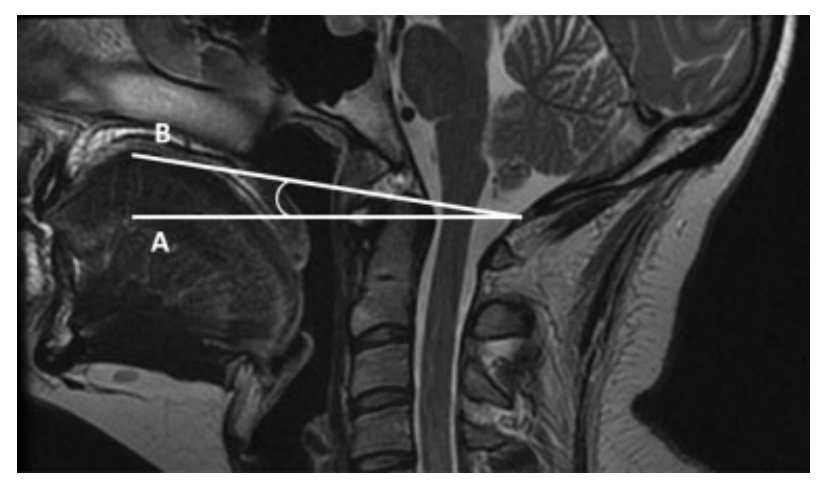

Fig. 1 Determination of angle of head flexion and extension on a midsagittal magnetic resonance imaging. Using electronic calipers, we first established the horizontal axial reference plane as defined by Karhu et al. ${ }^{4}$ The angle between this line (line A) and another line between the opisthion and the hard palate (line $B$ ) defined the extent of head flexion and extension, in degrees; line $B$ was above line $A$ in extension, they overlapped in a neutral position, and $B$ was below $A$ in flexion. 
the following parameters in the maximum flexion and extension positions achievable by patients (-Fig. 2 ):

- Cervicomedullary angle (CMA), which is the angle between two lines placed on the ventral aspects of the medulla and the cervical cord, respectively, determined on sagittal images ${ }^{4-6}$ (-Fig. 3); based on previous literature data, we considered a neutral head position CMA to be 140 degrees $^{4}$

- Displacement of the ventral surface of the brainstem, obtained by measuring the change in depth of the prepontine and premedullary cisterns perpendicular to the posterior clivus margin (-Fig. 4)

- Total sagittal surface area of the suprasellar, interpeduncular, prepontine and premedullary cisterns after tracing their outline

- Basilar tip to tuber cinereum distance

\section{Results}

The 15 patients ranged in age from 54 years to 72 years (mean 67 years); $63 \%$ were female and $37 \%$ male. A mean angle of -16 degrees (standard deviation [SD] of 8.5 degrees; range of -6 to -29 degrees) in maximum head extension was achieved in all 15 patients. A mean angle of +9.5 degrees
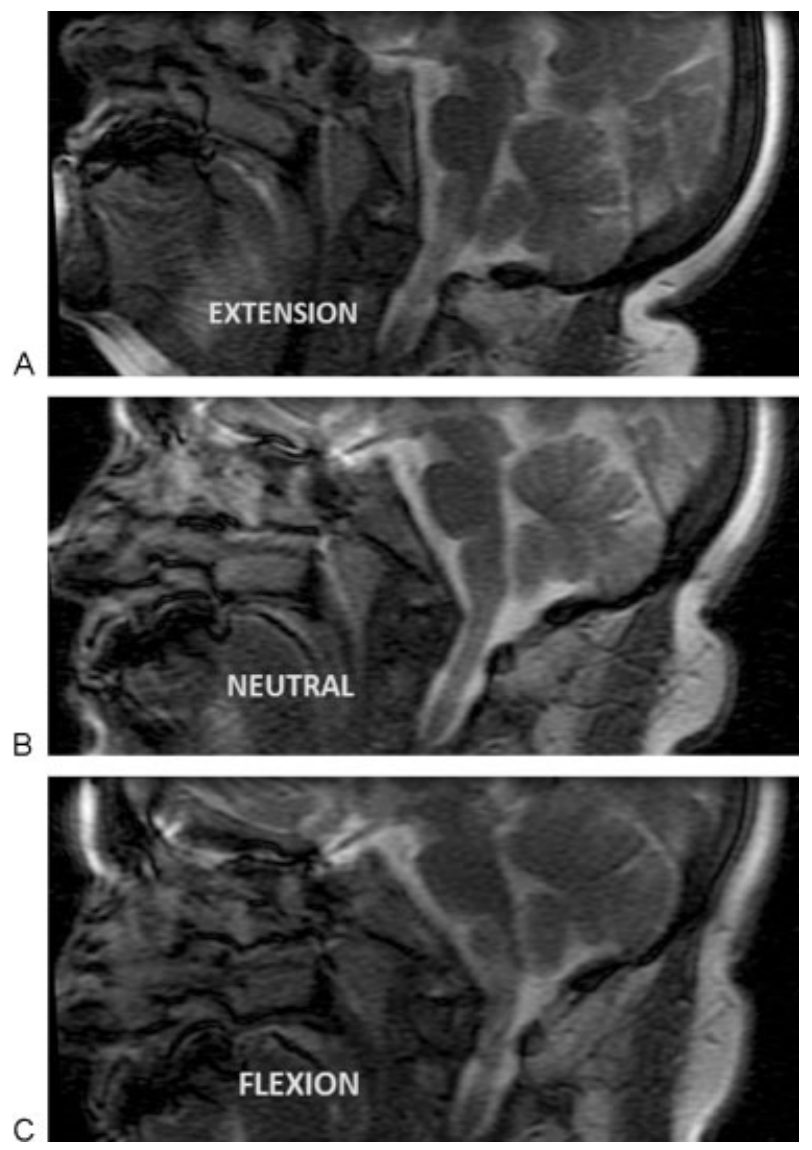

Fig. 2 Sequential still images from a kinematic magnetic resonance imaging of the basal head and upper cervical spine in the midsagittal plane showing head position as it changes from extension (A), to neutral position (B), to flexion (C).

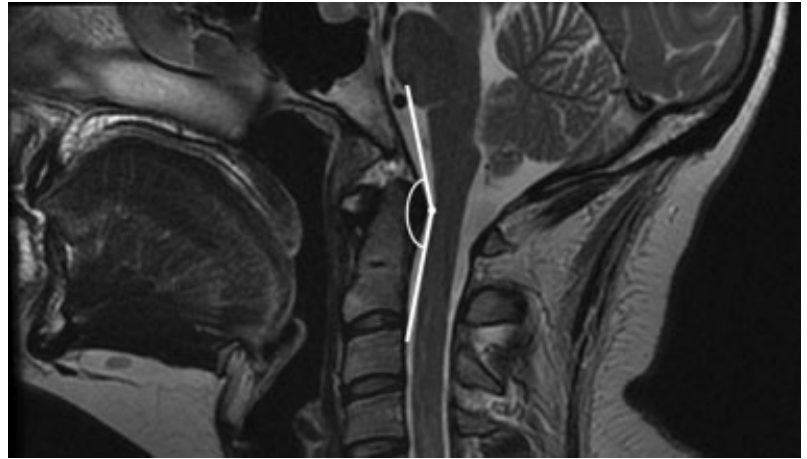

Fig. 3 Midsagittal magnetic resonance imaging showing the cervicomedullary angle, in degrees.

(SD of 0.8 degrees; range of +9.2 degrees to +11.2 degrees) in maximum head flexion was achieved in 6 patients. The remaining 9 patients were not able to flex their head past neutral.

The results are presented in detail as mean, standard deviation, and range, as displayed in -Table 1. The mean cervicomedullary angle was 158 degrees in maximum extension and 146 degrees in maximum flexion. We found mild displacement of the brainstem toward the clivus even on modest degrees of head flexion. The mean reduction in depth of the prepontine and premedullary cisterns, from maximum extension to maximum flexion, was $0.7 \mathrm{~mm}$ and $0.5 \mathrm{~mm}$, respectively. The combined area of suprasellar and ventral brainstem cisterns was minimally reduced from a mean of $402 \mathrm{~mm}^{2}$ in maximum head flexion to $399 \mathrm{~mm}^{2}$ in maximum extension. The basilar tip did not move significantly from its position in maximum extension to maximum flexion, a mean of $5.2 \mathrm{~mm}$ to $5.3 \mathrm{~mm}$ from the tuber cinereum, respectively.

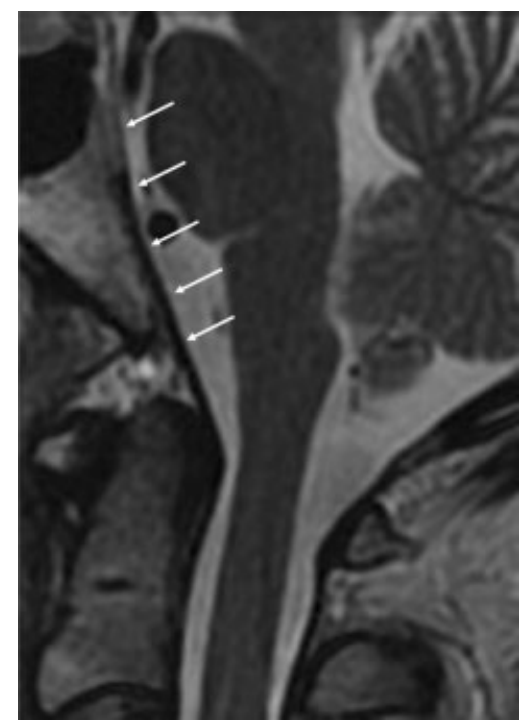

Fig. 4 Midsagittal magnetic resonance imaging showing direction of displacement (arrows) of the ventral surface of the brainstem relative to the clivus; modest flexion minimally alters the depth of the prepontine and premedullary cisterns. 
Table 1 The results are presented in detail as mean, standard deviation, and range for the parameters outlined

\begin{tabular}{|c|c|c|c|c|c|c|}
\hline & \multicolumn{3}{|c|}{$\begin{array}{l}\text { At maximum head extension } \\
\text { achieved in patients }(n=15)\end{array}$} & \multicolumn{3}{|c|}{$\begin{array}{l}\text { At maximum head flexion achieved } \\
\text { in patients }(n=6)\end{array}$} \\
\hline & mean & $\pm S D$ & range & mean & $\pm \mathrm{SD}$ & range \\
\hline Cervicomedullary angle (degrees) & 158 & 12.5 & $150-174$ & 146 & 7.8 & $132-150$ \\
\hline Prepontine cistern depth $(\mathrm{mm})$ & 4.8 & 2.2 & $2.3-9.0$ & 4.1 & 1.3 & $3.0-6.8$ \\
\hline Premedullary cistern depth (mm) & 5.6 & 0.9 & $3.7-6.8$ & 5.1 & 1.0 & $4.1-7.9$ \\
\hline $\begin{array}{l}\text { Total sagittal area of combined } \\
\text { suprasellar and ventral brainstem } \\
\text { cisterns }\left(\mathrm{mm}^{2}\right)\end{array}$ & 402 & 59 & $213-514$ & 399 & 62 & $277-454$ \\
\hline $\begin{array}{l}\text { Distance of basilar tip to tuber } \\
\text { cinereum ( } \mathrm{mm})\end{array}$ & 5.2 & 0.7 & $2.8-5.4$ & 5.3 & 0.8 & $3.2-6.3$ \\
\hline
\end{tabular}

Abbreviation: SD, standard deviation.

\section{Discussion}

Our results using kinematic MRI on subjects with no intracranial pathology show a very small displacement of caudal brain structures relative to the skull base within minor physiological changes in head position. To better understand these findings and to place them in further context, it would be useful for two main reasons to appreciate the results of other areas of research pertaining to brain motion: first, previous information might shed some light on whether our observed results may not be consequent to the head movement per se, but rather fall within the expected normal range of physiological pulsatile brain movement. Second, it would be useful to learn from previous studies of relative brain/skull displacement during low- or high-velocity impact consequent to head injuries.

Extremely low-velocity normal physiological motion may be expected in organs such as the brain. ${ }^{7,8}$ Arterial expansion causes the major part of such measured normal brain movements. ${ }^{7}$ Overall, solid brain tissue does not accumulate net displacement over a complete cardiac cycle. ${ }^{9-11}$ The centrifugal brain movements caused by normal brain expansion in systole are estimated to be less than $0.01 \mathrm{~mm}$ in magnitude and too small for detection on routine clinical MRI sequences. ${ }^{7}$ Indeed, it has been reported that the brain tissue movement within a cardiac cycle is only a small fraction of an image pixel. ${ }^{10}$ The measured displacements that we recorded (and those obtained by Ji et al, ${ }^{1}$ see below) upon variations in head movement were of considerably larger orders of magnitude than this reported physiological pulsatile brain movement. Therefore, our results are most likely entirely consequent to the head flexion and extension and cannot be ascribed instead to physiological brain displacements that occur normally during each cardiac cycle.

There has been a long-standing interest in the study of differential brain/skull displacement, especially when this might be significant or detrimental within the context of head trauma. The long-postulated local relative motion at the brain/skull interface was first directly observed by invasive means (e.g., replacing a portion of the macaque monkey skull with a transparent lucite calvarium and subsequent observations of brain displacement using high-speed cameras). ${ }^{12}$

To further investigate the local relative motion at brain/ skull interface, a more recent study by Ji et al described the relative displacement at the brain/skull boundary obtained noninvasively in five living humans under normal physiological conditions using kinematic MRI. ${ }^{1}$ This clearly revealed displacements of the normal brain relative to the skull as the head position changes from extension to flexion. The brainstems of all subjects moved toward the clivus upon flexion by mean distances ranging from $1.2 \mathrm{~mm}$ to $2.0 \mathrm{~mm}$. The cerebellum also moved in a caudad direction by mean distances ranging from $1.3 \mathrm{~mm}$ to $1.8 \mathrm{~mm}$. Our results showing submillimeter anterior movements of the brainstem upon head flexion concur with these findings of Ji et al, ${ }^{1}$ especially when due allowance is made for the much lower degrees of head flexion and extension achieved in our patient cohort. This limited neck movement was judged to be appropriate for our study because it better represents the modest movements that might be required for patient positioning prior to neuroendoscopic procedures, as opposed to extreme movements that would in theory be attainable in normal volunteers.

Taken together, this information gleaned from previous studies of normal pulsatile brain motion and from studies of differential brain/skull displacement upon slow or fast head movement can help clarify the results of our study. The relative brain/skull motion that we observed can be explained by the anatomic structures concerned and their biomechanical properties and behavior. The brain floats inside a pressurized cerebrospinal fluid (CSF)-filled space within the skull. ${ }^{1}$ Displacement of brain within the skull is resisted by the squeezing required to move CSF within the head. ${ }^{13}$ The magnitude of this resistance is proportional to the velocity of the head movement. ${ }^{13}$ Importantly, therefore, although CSF provides effective damping against sudden head motions (e.g., during impact at head trauma), the damping effect decreases when the head undergoes slow motion. ${ }^{1}$ Under these low CSF resistance conditions, as may be encountered during slow normal physiological head movements or when placing an anesthetized patient in a particular head position, the brain tends to sink within the intracranial cavity along the 
direction of gravity, ${ }^{1}$ as revealed by our imaging findings. This arises on account of the mass density of the brain tissue (1.04 to $\left.1.05 \mathrm{~kg} / \mathrm{m}^{3}\right)^{1,13}$ being slightly greater than that of CSF (1.004 to $\left.1.007 \mathrm{~kg} / \mathrm{m}^{3}\right)^{1,14}$

In conclusion, our results demonstrate that upon kinematic analysis of brain MRI in the elderly, relative basal brain/ skull displacements are very small and in the order of $<1 \mathrm{~mm}$ when the head position changes from modest extension to modest flexion. Potential intracranial displacements in children or that might arise after more extreme head flexion and extension would need to be investigated in a separate prospective analysis of normal volunteers. The minimal brain displacements observed in our study are much too small to be considered as factors that, in themselves, could significantly affect decisions regarding patient positioning for neuroendoscopic procedure. This clearly does not alter the necessary general caution applicable to operative patient positioning during open craniotomy that may inadvertently result in cervical myelopathies or brachial plexopathies (and, for example, that might be detected using intraoperative somatosensory evoked potential monitoring), ${ }^{15}$ and which may also be relevant to head positioning of an anesthetized patient during neuroendoscopy. Our results suggest that, unlike the occasional extracranial neural compression that might occur during neurosurgical procedures, the normal brainstem above the foramen magnum might be unaffected by any operative position-induced modest displacements of the head.

Reassuringly, there is no significant movement of the basilar tip in modest flexion and extension to affect fenestration of the tuber cinereum during endoscopic third ventriculostomy. This is critical, since injury to the basilar tip or its branches is a serious complication of this procedure. ${ }^{16}$ Knowing that the position of the basilar tip relative to the tuber cinereum is independent of the patient's head position is valuable information for the neuroendoscopist. Likewise, the minimal anterior movement of the brainstem toward the clivus in flexion is unlikely to affect trajectories during the expanded endonasal endoscopic approach to the retroclival space $^{17}$ or distinct anatomical targets within it. ${ }^{18,19}$ It is also encouraging to learn that the small degree of patient head flexion advocated by Jho and Ha during endoscopic endonasal transsphenoidal approaches to the clivus and posterior fossa do not significantly change the position of the brainstem relative to the clivus. ${ }^{20}$ Moreover, in situations where the ventral brainstem cisterns may already be effaced to some extent by a large mass requiring neuroendoscopic debulking (e.g., a clival chordoma), ${ }^{21}$ it is also reassuring that modest patient head flexion desirable for this procedure is not likely in theory to aggravate any preexisting brainstem compression. Prior reports have demonstrated that the patient's head position during preoperative imaging plays no relevant role in intraoperative accuracy of neuronavigation. ${ }^{22}$ Our comparable results using kinematic MRI showing no significant movement of basal brain structures should be useful in presurgical planning of optimal patient positioning for neuroendoscopic procedures.

\section{Acknowledgment}

T.F.M. was supported by the NIHR Cambridge Biomedical Research Center.

\section{Conflict of Interest \\ None}

\section{References}

1 Ji S, Zhu Q, Dougherty L, Margulies SS. In vivo measurements of human brain displacement. Stapp Car Crash J 2004;48:227-237

2 Lawlor DA, Stone T. Public health and data protection: an inevitable collision or potential for a meeting of minds? Int J Epidemiol 2001;30:1221-1225

3 Schroter S, Plowman R, Hutchings A, Gonzalez A. Reporting ethics committee approval and patient consent by study design in five general medical journals. J Med Ethics 2006;32:718-723

4 Karhu JO, Parkkola RK, Koskinen SK. Evaluation of flexion/extension of the upper cervical spine in patients with rheumatoid arthritis: an MRI study with a dedicated positioning device compared to conventional radiographs. Acta Radiol 2005;46:5566

5 Reijnierse M, Breedveld FC, Kroon HM, Hansen B, Pope TL, Bloem JL. Are magnetic resonance flexion views useful in evaluating the cervical spine of patients with rheumatoid arthritis? Skeletal Radiol 2000;29:85-89

6 Bundschuh C, Modic MT, Kearney F, Morris R, Deal C. Rheumatoid arthritis of the cervical spine: surface-coil MR imaging. AJR Am J Roentgenol 1988;151:181-187

7 Greitz D, Wirestam R, Franck A, Nordell B, Thomsen C, Ståhlberg F. Pulsatile brain movement and associated hydrodynamics studied by magnetic resonance phase imaging. The Monro-Kellie doctrine revisited. Neuroradiology 1992;34:370-380

8 Feinberg DA, Mark AS. Human brain motion and cerebrospinal fluid circulation demonstrated with MR velocity imaging. Radiology 1987;163:793-799

9 Zhu DC, Xenos M, Linninger AA, Penn RD. Dynamics of lateral ventricle and cerebrospinal fluid in normal and hydrocephalic brains. J Magn Reson Imaging 2006;24:756-770

10 Enzmann DR, Pelc NJ. Brain motion: measurement with phasecontrast MR imaging. Radiology 1992;185:653-660

11 Poncelet BP, Wedeen VJ, Weisskoff RM, Cohen MS. Brain parenchyma motion: measurement with cine echo-planar MR imaging. Radiology 1992;185:645-651

12 Pudenz RH, Shelden CH. The lucite calvarium; a method for direct observation of the brain; cranial trauma and brain movement. J Neurosurg 1946;3:487-505

13 Couper Z, Albermani F. Infant brain subjected to oscillatory loading: material differentiation, properties, and interface conditions. Biomech Model Mechanobiol 2008;7:105-125

14 Belingardi G, Chiandussi G, Gaviglio I. Development and validation of a new finite element model of human head. Available at: http:// www.biomedcafeonline.com/documents/aow9.pdf. Accessed June 25, 2012

15 Anastasian ZH, Ramnath B, Komotar RJ, et al. Evoked potential monitoring identifies possible neurological injury during positioning for craniotomy. Anesth Analg 2009;109:817-821

16 Fabiano AJ, Leonardo J, Grand W. Posterior cerebral artery P1 segment at the stoma during endoscopic third ventriculostomy in adults. J Neurol Neurosurg Psychiatry 2010;81:374-378

17 Kassam A, Snyderman CH, Mintz A, Gardner P, Carrau RL. Expanded endonasal approach: the rostrocaudal axis. Part II. Posterior clinoids to the foramen magnum. Neurosurg Focus 2005;19:E4 
18 Prevedello DM, Ditzel Filho LF, Solari D, Carrau RL, Kassam AB. Expanded endonasal approaches to middle cranial fossa and posterior fossa tumors. Neurosurg Clin N Am 2010;21:621635, vi

19 Kassam AB, Mintz AH, Gardner PA, Horowitz MB, Carrau RL, Snyderman $\mathrm{CH}$. The expanded endonasal approach for an endoscopic transnasal clipping and aneurysmorrhaphy of a large vertebral artery aneurysm: technical case report. Neurosurgery 2006;59(1, Suppl 1):E162-E165, discussion E162-E165
20 Jho HD, Ha HG. Endoscopic endonasal skull base surgery: Part 3The clivus and posterior fossa. Minim Invasive Neurosurg 2004;47: 16-23

21 Holzmann D, Reisch R, Krayenbühl N, Hug E, Bernays RL. The transnasal transclival approach for clivus chordoma. Minim Invasive Neurosurg 2010;53:211-217

22 Reinges $\mathrm{MH}$, Krings T, Nguyen $\mathrm{HH}$, et al. Is the head position during preoperative image data acquisition essential for the accuracy of navigated brain tumor surgery? Comput Aided Surg 2000;5:426-432 